\title{
ON EXISTENCE OF A CLASSICAL SOLUTION AND NON-EXISTENCE OF A WEAK SOLUTION TO THE DIRICHLET PROBLEM IN A PLANAR DOMAIN WITH SLITS FOR LAPLACIAN
}

BY

\author{
P. A. KRUTITSKII
}

KIAM, Department 25, Miusskaya Sq. 4, Moscow 125047, Russia

\begin{abstract}
The Dirichlet problem for the Laplacian in a planar domain bounded by smooth closed curves and smooth double-sided open arcs (slits) is considered in the case when the solution is not continuous at the ends of the slits. The cases of both interior and exterior domains are considered. The well-posed formulation of the problem is given, theorems on existence and uniqueness of a classical solution are proved, and the integral representation for a solution is obtained. It is shown that a weak solution of the problem does not typically exist, though the classical solution exists.
\end{abstract}

1. Introduction. Boundary value problems in planar domains with slits (doublesided open arcs) are very important for applications, since slits model cracks, screens, wings, and other thin objects in physics, in mechanics, and in engineering [1].

It is known that if the Dirichlet problem for the Laplacian is considered in a planar domain bounded by sufficiently smooth closed curves, and if the function specified in the boundary condition is smooth enough, then existence of a classical solution follows from existence of a weak solution. In the present paper we consider the Dirichlet problem for the Laplacian in both interior and exterior planar domains bounded by closed curves and double-sided open arcs (slits) of an arbitrary shape. The Dirichlet condition is specified on the whole boundary, i.e., on both closed curves and on the slits, so that different functions may be specified on opposite sides of the slits. The case of this problem, when the solution is continuous at the tips of the slits, has been previously studied in [2]--[6], where theorems on existence and uniqueness of a classical solution have been proved and the integral representation for a classical solution has been obtained. In the present paper, this problem is considered in the case when the solution may be not continuous at the tips of the slits. We prove that there exists a unique classical solution to this problem and obtain an integral representation for the classical solution. In addition, we prove that a weak solution to this problem may not exist even if both slits and functions in the boundary conditions are sufficiently smooth. This result follows from the fact that

Received April 6, 2007.

2000 Mathematics Subject Classification. Primary 35J05, 35J25.

(C)2007 Brown University 
the square of the gradient of a classical solution, basically, is not integrable near the ends of the slits, since singularities of the gradient are rather strong there. This result is very important for numerical analysis; it shows that finite elements and finite difference methods cannot be applied to numerical treatment of the Dirichlet problem in question directly, since all of these methods imply existence of a weak solution. To use difference methods for numerical analysis, one has to localize all strong singularities first and then use difference methods in a domain excluding the neighbourhoods of the singularities.

2. Formulation of the problem. By an open curve we mean a simple smooth non-closed arc of finite length without self-intersections [7].

Let $\Gamma$ be a set of curves, which may be both closed and open. We say that $\Gamma \in C^{2, \lambda}$ (or $\Gamma \in C^{1, \lambda}$ ) if the curves $\Gamma$ belong to the class $C^{2, \lambda}$ (or $C^{1, \lambda}$ ) with the Hölder exponent $\lambda \in(0,1]$.

In a plane in Cartesian coordinates $x=\left(x_{1}, x_{2}\right) \in R^{2}$ we consider a multiply connected domain bounded by simple open curves $\Gamma_{1}^{1}, \ldots, \Gamma_{N_{1}}^{1} \in C^{2, \lambda}$ and simple closed curves $\Gamma_{1}^{2}, \ldots, \Gamma_{N_{2}}^{2} \in C^{2, \lambda}, \lambda \in(0,1]$, in such a way that all curves do not have common points, in particular, endpoints. We will consider both the case of an exterior domain and the case of an interior domain when the curve $\Gamma_{1}^{2}$ encloses all others. Set

$$
\Gamma^{1}=\bigcup_{n=1}^{N_{1}} \Gamma_{n}^{1}, \quad \Gamma^{2}=\bigcup_{n=1}^{N_{2}} \Gamma_{n}^{2}, \quad \Gamma=\Gamma^{1} \cup \Gamma^{2} .
$$

The connected domain bounded by $\Gamma^{2}$ and containing curves $\Gamma^{1}$ will be called $\mathcal{D}$, so that $\partial \mathcal{D}=\Gamma^{2}, \Gamma^{1} \subset \mathcal{D}$. We assume that each curve $\Gamma_{n}^{j}$ is parametrized by the arc length $s$ :

$$
\Gamma_{n}^{j}=\left\{x: \quad x=x(s)=\left(x_{1}(s), x_{2}(s)\right), \quad s \in\left[a_{n}^{j}, b_{n}^{j}\right]\right\}, \quad n=1, \ldots, N_{j}, \quad j=1,2,
$$

so that

$$
a_{1}^{1}<b_{1}^{1}<\cdots<a_{N_{1}}^{1}<b_{N_{1}}^{1}<a_{1}^{2}<b_{1}^{2}<\cdots<a_{N_{2}}^{2}<b_{N_{2}}^{2},
$$

and the domain $\mathcal{D}$ is placed to the right when the parameter $s$ increases on $\Gamma_{n}^{2}$. The points $x \in \Gamma$ and values of the parameter $s$ are in one-to-one correspondence except the points $a_{n}^{2}, b_{n}^{2}$, which correspond to the same point $x$ for $n=1, \ldots, N_{2}$. Further on, the set of the intervals

$$
\bigcup_{n=1}^{N_{1}}\left[a_{n}^{1}, b_{n}^{1}\right], \quad \bigcup_{n=1}^{N_{2}}\left[a_{n}^{2}, b_{n}^{2}\right], \quad \bigcup_{j=1}^{2} \bigcup_{n=1}^{N_{j}}\left[a_{n}^{j}, b_{n}^{j}\right]
$$

on the $O s$-axis will be denoted by $\Gamma^{1}, \Gamma^{2}$, and $\Gamma$ also.

$\operatorname{Set} C^{j, r}\left(\Gamma_{n}^{2}\right)=\left\{\mathcal{F}(s): \mathcal{F}(s) \in C^{j, r}\left[a_{n}^{2}, b_{n}^{2}\right], \mathcal{F}^{(m)}\left(a_{n}^{2}\right)=\mathcal{F}^{(m)}\left(b_{n}^{2}\right), m=0, \ldots, j\right\}$, $j=0,1, r \in[0,1]$ and $C^{j, r}\left(\Gamma^{2}\right)=\bigcap_{n=1}^{N_{2}} C^{j, r}\left(\Gamma_{n}^{2}\right)$. The tangent vector to $\Gamma$ in the point $x(s)$, in the direction of the increment of $s$, will be denoted by $\tau_{x}=(\cos \alpha(s), \sin \alpha(s))$, while the normal vector coinciding with $\tau_{x}$ after rotation through an angle of $\pi / 2$ in the counterclockwise direction will be denoted by $\mathbf{n}_{x}=(\sin \alpha(s),-\cos \alpha(s))$. According to the chosen parametrization $\cos \alpha(s)=x_{1}^{\prime}(s), \sin \alpha(s)=x_{2}^{\prime}(s)$. Thus, $\mathbf{n}_{x}$ is an interior 
normal to $\mathcal{D}$ on $\Gamma^{2}$. By $X$ we denote the point set consisting of the endpoints of $\Gamma^{1}$ : $X=\bigcup_{n=1}^{N_{1}}\left(x\left(a_{n}^{1}\right) \cup x\left(b_{n}^{1}\right)\right)$.

Let the plane be slit along $\Gamma^{1}$. We consider $\Gamma^{1}$ as a set of slits (or double-sided open arcs). The side of the slit $\Gamma^{1}$, which is situated on the left when the parameter $s$ increases, will be denoted by $\left(\Gamma^{1}\right)^{+}$, while the opposite side will be denoted by $\left(\Gamma^{1}\right)^{-}$.

We say that the function $u(x)$ belongs to the smoothness class $\mathbf{K}_{1}$ if

(1) $u \in C^{0}\left(\overline{\mathcal{D} \backslash \Gamma^{1}} \backslash X\right) \cap C^{2}\left(\mathcal{D} \backslash \Gamma^{1}\right), \quad \nabla u \in C^{0}\left(\overline{\mathcal{D} \backslash \Gamma^{1}} \backslash \Gamma^{2} \backslash X\right)$,

(2) in the neighbourhood of any point $x(d) \in X$, the equality

$$
\lim _{r \rightarrow+0} \int_{\partial S(d, r)} u(x) \frac{\partial u(x)}{\partial \mathbf{n}_{x}} d l=0
$$

holds, where the curvilinear integral of the first kind is taken over a circumference $\partial S(d, r)$ of a radius $r$ with the center in the point $x(d)$. In addition, $\mathbf{n}_{x}$ is a normal in the point $x \in \partial S(d, r)$, directed to the center of the circumference and $d=a_{n}^{1}$ or $d=b_{n}^{1}, n=1, \ldots, N_{1}$.

Remark. By $C^{0}\left(\overline{\mathcal{D} \backslash \Gamma^{1}} \backslash X\right)$ we denote the class of continuous in $\overline{\mathcal{D}} \backslash \Gamma^{1}$ functions that are continuously extensible to the sides of the slits $\Gamma^{1} \backslash X$ from the left and from the right, but their limiting values on $\Gamma^{1} \backslash X$ can be different from the left and from the right, so that these functions may have a jump on $\Gamma^{1} \backslash X$. To obtain the definition of the class $C^{0}\left(\overline{\mathcal{D} \backslash \Gamma^{1}} \backslash \Gamma^{2} \backslash X\right)$ we have to replace $C^{0}\left(\overline{\mathcal{D} \backslash \Gamma^{1}} \backslash X\right)$ by $C^{0}\left(\overline{\mathcal{D} \backslash \Gamma^{1}} \backslash \Gamma^{2} \backslash X\right)$ and $\overline{\mathcal{D}} \backslash \Gamma^{1}$ by $\mathcal{D} \backslash \Gamma^{1}$ in the previous sentence.

Let us formulate the Dirichlet problem for the Laplacian in a domain $\mathcal{D} \backslash \Gamma^{1}$ (interior or exterior).

Problem $\mathbf{D}_{1}$. Find a function $u(x)$ from the class $\mathbf{K}_{1}$ so that $u(x)$ obeys the Laplace equation

$$
u_{x_{1} x_{1}}(x)+u_{x_{2} x_{2}}(x)=0
$$

in $\mathcal{D} \backslash \Gamma^{1}$ and satisfies the boundary conditions

$$
\left.u(x)\right|_{x(s) \in\left(\Gamma^{1}\right)^{+}}=F^{+}(s),\left.\quad u(x)\right|_{x(s) \in\left(\Gamma^{1}\right)^{-}}=F^{-}(s),\left.\quad u(x)\right|_{x(s) \in \Gamma^{2}}=F(s) .
$$

If $\mathcal{D}$ is an exterior domain, then we add the following condition at infinity:

$$
|u(x)| \leq \text { const, } \quad|x|=\sqrt{x_{1}^{2}+x_{2}^{2}} \rightarrow \infty .
$$

All conditions of the problem $\mathbf{D}_{1}$ must be satisfied in a classical sense. The boundary conditions $(2 \mathrm{~b})$ on $\Gamma^{1}$ must be satisfied in the interior points of $\Gamma^{1}$; their validity at the ends of $\Gamma^{1}$ is not required.

Theorem 1. If $\Gamma \in C^{2, \lambda}, \lambda \in(0,1]$, then there is no more than one solution to the problem $\mathbf{D}_{1}$.

Proof. It is sufficient to prove that the homogeneous problem $\mathbf{D}_{1}$ admits the trivial solution only. Let $u^{0}(x)$ be a solution to the homogeneous problem $\mathbf{D}_{1}$ with $F^{+}(s) \equiv$ $F^{-}(s) \equiv 0, F(s) \equiv 0$. Let $S(d, \epsilon)$ be a disc of a small enough radius $\epsilon$ with the center in the point $x(d) \quad\left(d=a_{n}^{1}\right.$ or $\left.d=b_{n}^{1}, n=1, \ldots, N_{1}\right)$. Let $\Gamma_{n, \epsilon}^{1}$ be a set consisting of such 
points of the curve $\Gamma_{n}^{1}$ which do not belong to discs $S\left(a_{n}^{1}, \epsilon\right)$ and $S\left(b_{n}^{1}, \epsilon\right)$. We choose a number $\epsilon_{0}$ so small that the following conditions are satisfied:

1) for any $0<\epsilon \leq \epsilon_{0}$ the set of points $\Gamma_{n, \epsilon}^{1}$ is a unique non-closed arc for each $n=1, \ldots, N_{1}$,

2) the points belonging to $\Gamma \backslash \Gamma_{n}^{1}$ are placed outside the discs $S\left(a_{n}^{1}, \epsilon_{0}\right), S\left(b_{n}^{1}, \epsilon_{0}\right)$ for any $n=1, \ldots, N_{1}$,

3) discs of radius $\epsilon_{0}$ with centers at different ends of $\Gamma^{1}$ do not intersect.

Set $\Gamma^{1, \epsilon}=\cup_{n=1}^{N_{1}} \Gamma_{n, \epsilon}^{1}, \quad S_{\epsilon}=\left(\cup_{n=1}^{N_{1}}\left[S\left(a_{n}^{1}, \epsilon\right) \cup S\left(b_{n}^{1}, \epsilon\right)\right]\right), \mathcal{D}_{\epsilon}=\mathcal{D} \backslash \Gamma^{1, \epsilon} \backslash S_{\epsilon}$. If $\mathcal{D}$ is an exterior domain, then we set $\mathcal{D}_{\epsilon, R}=\mathcal{D}_{\epsilon} \cap S^{R}$, where $S^{R}$ is a disc with a center in the origin and with sufficiently large radius $R$.

Since $\Gamma^{2} \in C^{2, \lambda}, u^{0}(x) \in C^{0}\left(\overline{\mathcal{D}} \backslash \Gamma^{1}\right.$ ) (remember that $u^{0}(x) \in \mathbf{K}_{1}$ ), and since $\left.u^{0}\right|_{\Gamma^{2}}=0 \in C^{2, \lambda}\left(\Gamma^{2}\right)$, and owing to the lemma on regularity of solutions of elliptic equations near the boundary [10, Lemma 6.18], we obtain $u^{0}(x) \in C^{1}\left(\overline{\mathcal{D}} \backslash \Gamma^{1}\right)$. Since $u^{0}(x) \in \mathbf{K}_{1}$, we observe that $u^{0}(x) \in C^{1}\left(\overline{\mathcal{D}}_{\epsilon}\right)$ for any $\epsilon \in\left(0, \epsilon_{0}\right]$. By $C^{1}\left(\overline{\mathcal{D}}_{\epsilon}\right)$ we mean $C^{1}\left(\mathcal{D}_{\epsilon} \cup \Gamma^{2} \cup\left(\Gamma^{1, \epsilon}\right)^{+} \cup\left(\Gamma^{1, \epsilon}\right)^{-} \cup \partial S_{\epsilon}\right)$. Analogously, in the case of an exterior domain $\mathcal{D}, u^{0}(x) \in C^{1}\left(\overline{\mathcal{D}}_{\epsilon, R}\right)$ for $\epsilon \in\left(0, \epsilon_{0}\right]$. Let $\mathcal{D}$ be an interior domain. Since the boundary of a domain $\mathcal{D}_{\epsilon}$ is piecewise smooth, we write out Green's formula [9, p. 328] for the function $u^{0}(x)$ :

$$
=\int_{\Gamma^{1, \epsilon}}\left(u^{0}\right)^{+}\left(\frac{\partial u^{0}}{\partial \mathbf{n}_{x}}\right)^{+} d s-\int_{\Gamma^{1, \epsilon}}\left(u^{0} \|^{0}{ }_{L_{2}\left(\mathcal{D}_{\epsilon}\right)}^{-}\left(\frac{\partial u^{0}}{\partial \mathbf{n}_{x}}\right)^{-} d s-\int_{\Gamma^{2}} u^{0} \frac{\partial u^{0}}{\partial \mathbf{n}_{x}} d s+\int_{\partial S_{\epsilon}} u^{0} \frac{\partial u^{0}}{\partial \mathbf{n}_{x}} d l .\right.
$$

We denote by $\mathbf{n}_{x}$ the exterior (with respect to $\mathcal{D}_{\epsilon}$ ) normal on $\partial S_{\epsilon}$ at the point $x \in \partial S_{\epsilon}$. By the superscripts + and - we denote the limiting values of functions on $\left(\Gamma^{1}\right)^{+}$and on $\left(\Gamma^{1}\right)^{-}$respectively. Since $u^{0}(x)$ satisfies the homogeneous boundary condition $(2 \mathrm{~b})$ on $\Gamma$, we observe that $\left.u^{0}\right|_{\Gamma^{2}}=0$ and $\left.\left(u^{0}\right)^{ \pm}\right|_{\Gamma^{1, \epsilon}}=0$ for any $\epsilon \in\left(0, \epsilon_{0}\right]$. Therefore identity (3) takes the form

$$
\left\|\nabla u^{0}\right\|_{L_{2}\left(\mathcal{D}_{\epsilon}\right)}^{2}=\int_{\partial S_{\epsilon}} u^{0} \frac{\partial u^{0}}{\partial \mathbf{n}_{x}} d l, \quad \epsilon \in\left(0, \epsilon_{0}\right] .
$$

Setting $\epsilon \rightarrow+0$ in (4), taking into account that $u^{0}(x) \in \mathbf{K}_{1}$ and using relationship (1), we obtain:

$$
\left\|\nabla u^{0}\right\|_{L_{2}\left(\mathcal{D} \backslash \Gamma^{1}\right)}^{2}=\lim _{\epsilon \rightarrow+0}\left\|\nabla u^{0}\right\|_{L_{2}\left(\mathcal{D}_{\epsilon}\right)}^{2}=0 .
$$

From the homogeneous boundary conditions $(2 \mathrm{~b})$ we conclude that $u^{0}(x) \equiv 0$ in $\mathcal{D} \backslash \Gamma^{1}$, where $\mathcal{D}$ is an interior domain.

Let $\mathcal{D}$ be an exterior domain. Since the boundary of a domain $\mathcal{D}_{\epsilon, R}$ is piecewise smooth and since $u^{0}(x) \in C^{1}\left(\overline{\mathcal{D}}_{\epsilon, R}\right)$ for any $\epsilon \in\left(0, \epsilon_{0}\right]$, we may write Green's formula in a domain $\mathcal{D}_{\epsilon, R}$ for a harmonic function $u^{0}(x)$ [9, p. 328]:

$$
\begin{gathered}
\left\|\nabla u^{0}\right\|_{L_{2}\left(\mathcal{D}_{\epsilon, R}\right)}^{2}=\int_{\Gamma^{1, \epsilon}}\left(u^{0}\right)^{+}\left(\frac{\partial u^{0}}{\partial \mathbf{n}_{x}}\right)^{+} d s-\int_{\Gamma^{1, \epsilon}}\left(u^{0}\right)^{-}\left(\frac{\partial u^{0}}{\partial \mathbf{n}_{x}}\right)^{-} d s \\
-\int_{\Gamma^{2}} u^{0} \frac{\partial u^{0}}{\partial \mathbf{n}_{x}} d s+\int_{\partial S_{\epsilon}} u^{0} \frac{\partial u^{0}}{\partial \mathbf{n}_{x}} d l+\int_{\partial S^{R}} u^{0} \frac{\partial u^{0}}{\partial|x|} d l .
\end{gathered}
$$


By $\mathbf{n}_{x}$ on $\partial S_{\epsilon}$ we denote an outward (with respect to $\mathcal{D}_{\epsilon, R}$ ) normal in the point $x \in \partial S_{\epsilon}$. It follows from condition (2c) and from the theorem on behaviour of a gradient of a harmonic function at infinity $[9$, p. 373] that

$$
\frac{\partial u^{0}(x)}{\partial|x|}=O\left(\frac{1}{|x|^{2}}\right), \quad \text { as } \quad|x| \rightarrow \infty .
$$

Consequently,

$$
\lim _{R \rightarrow \infty} \int_{\partial S^{R}} u^{0}(x) \frac{\partial u^{0}(x)}{\partial|x|} d l=0
$$

and formula (6) transforms to the formula (3) as $R \rightarrow \infty$. Repeating all arguments as presented above for the case of an interior domain $\mathcal{D}$, we arrive at formula (5). Taking into account homogeneous boundary conditions $(2 \mathrm{~b})$, we obtain from (5) that $u^{0}(x) \equiv 0$ in $\mathcal{D} \backslash \Gamma^{1}$, where $\mathcal{D}$ is an exterior domain. Thus, in all cases $u^{0}(x) \equiv 0$ in $\mathcal{D} \backslash \Gamma^{1}$. The theorem is proved.

REMARK. The maximum principle cannot be used for the proof of the theorem even in the case of an interior domain $\mathcal{D}$, since the solution to the problem may not satisfy the boundary condition $(2 \mathrm{~b})$ at the ends of the slits, and it may not be continuous at the ends of the slits.

3. Properties of the double layer potential on the open curve. Let $\gamma$ be an open curve of class $C^{1, \lambda}, \quad \lambda \in(0,1]$. Assume that $\gamma$ is parametrized by the arc length $s: \quad \gamma=\left\{x: x(s)=\left(x_{1}(s), x_{2}(s)\right), s \in[a, b]\right\}$. The points $x \in \Gamma$ and values of the parameter $s$ are in one-to-one correspondence, so the segment $[a, b]$ will be also denoted by $\gamma$. The tangent vector to $\gamma$ in the point $x(s)$, in the direction of the increment of $s$, will be denoted by $\tau_{x}=(\cos \alpha(s), \sin \alpha(s))$, while the normal vector to $\gamma$ in the point $x(s)$ will be denoted by $\mathbf{n}_{x}=(\sin \alpha(s),-\cos \alpha(s))$. According to the chosen parametrization $\cos \alpha(s)=x_{1}^{\prime}(s), \quad \sin \alpha(s)=x_{2}^{\prime}(s)$. Let the plane be slit along $\gamma$. The side of the slit $\gamma$, which is situated on the left when the parameter $s$ increases, will be denoted by $\gamma^{+}$, while the opposite side will be denoted by $\gamma^{-}$. Let $X_{\gamma}=x(a) \cup x(b)$ be a set of the ends of $\gamma$.

Set $\mu(s) \in C^{0, \lambda}[a, b]$, and consider the double layer potential for the Laplacian in a plane

$$
\mathcal{W}[\mu](x)=-\frac{1}{2 \pi} \int_{a}^{b} \mu(\sigma) \frac{\partial}{\partial \mathbf{n}_{y}} \ln |x-y(\sigma)| d \sigma .
$$

Set $z=x_{1}+i x_{2}, \quad t=t(\sigma)=\left(y_{1}(\sigma)+i y_{2}(\sigma)\right) \in \gamma, \quad \hat{\mu}(t)=\hat{\mu}(t(\sigma))=\mu(\sigma)$. If $\mu(s) \in$ $C^{0, \lambda}[a, b]$, then $\hat{\mu}(t) \in C^{0, \lambda}(\gamma)$, since

$$
\begin{gathered}
\left|\hat{\mu}\left(t_{2}\right)-\hat{\mu}\left(t_{1}\right)\right|=\left|\hat{\mu}\left(t\left(\sigma_{2}\right)\right)-\hat{\mu}\left(t\left(\sigma_{1}\right)\right)\right|=\left|\mu\left(\sigma_{2}\right)-\mu\left(\sigma_{1}\right)\right| \leq c\left|\sigma_{2}-\sigma_{1}\right|^{\lambda} \\
=c\left(\frac{\left|\sigma_{2}-\sigma_{1}\right|}{\left|t\left(\sigma_{2}\right)-t\left(\sigma_{1}\right)\right|}\right)^{\lambda}\left|t\left(\sigma_{2}\right)-t\left(\sigma_{1}\right)\right|^{\lambda}=c \cdot c_{0}^{\lambda}\left|t_{2}-t_{1}\right|^{\lambda},
\end{gathered}
$$

where $c$ and $c_{0}$ are constants, $t_{2}=t\left(\sigma_{2}\right) \in \gamma, t_{1}=t\left(\sigma_{1}\right) \in \gamma$. We took into account in deriving the latter inequality that

$$
\frac{\left|\sigma_{2}-\sigma_{1}\right|}{\left|t\left(\sigma_{2}\right)-t\left(\sigma_{1}\right)\right|} \in C^{0}([a, b] \times[a, b])
$$


(see Lemma 1 in [11]), whence

$$
\frac{\left|\sigma_{2}-\sigma_{1}\right|}{\left|t\left(\sigma_{2}\right)-t\left(\sigma_{1}\right)\right|} \leq c_{0} .
$$

Consider an integral of Cauchy type with real density $\hat{\mu}(t)$ :

$$
\Phi(z)=\frac{1}{2 \pi i} \int_{\gamma} \hat{\mu}(t) \frac{d t}{t-z}
$$

then $\mathcal{W}[\mu](x)=-\operatorname{Re} \Phi(z)$. It follows from properties of a Cauchy-type integral that if $\mu(\sigma) \in C^{0, \lambda}[a, b]$, then $\mathcal{W}[\mu](x) \in C^{0}\left(\overline{R^{2} \backslash \gamma} \backslash X_{\gamma}\right)$. This means that the potential $\mathcal{W}[\mu](x)$ is continuously extensible to $\gamma$ from the left and from the right in interior points (though its values on $\gamma$ from the left and from the right may be different). If, in addition, $\mu(d)=0$, then the potential $\mathcal{W}[\mu](x)$ is continuously extensible to the end $x(d)$, where $d=a$ or $d=b \quad($ see $[7, \S 15.2])$. Set

$$
\cos \psi(x, y)=\frac{x_{1}-y_{1}}{|x-y|}=-|x-y|_{y_{1}}^{\prime}, \quad \sin \psi(x, y)=\frac{x_{2}-y_{2}}{|x-y|}=-|x-y|_{y_{2}}^{\prime} .
$$

Then $\psi(x, y)$ is a polar angle of the coordinate system with the origin in the point $y$. Formulae for $\cos \psi(x, y), \sin \psi(x, y)$ define the angle $\psi(x, y)$ with indeterminacy up to $2 \pi m$ ( $m$ being an integer). Let $S(d, \epsilon)$ be a disc of a sufficiently small radius $\epsilon$ with the center in $x(d) \quad(d=a$ or $d=b)$. From asymptotic formulae describing $\Phi(z)$ at the ends of $\gamma[7, \S 22]$, we may derive the asymptotic formulae for $\mathcal{W}[\mu](x)=-\operatorname{Re} \Phi(z)$ at the ends of $\gamma$. Namely, for any $x \in S(d, \epsilon)$ and $x \notin \gamma$, the formulae holds:

$$
\mathcal{W}[\mu](x)=\frac{ \pm \mu(d)}{2 \pi} \psi(x, x(d))+\Omega(x) .
$$

Here by $\psi(x, x(d))$ we mean some fixed branch of this function, so that the branch varies continuously in $x$ in a neighbourhood of the point $x(d)$, slit along $\gamma$. The upper sign is taken if $d=a$, while the lower sign is taken if $d=b$. The function $\Omega(x)$ is continuous as $x \rightarrow x(d)$. Moreover, $\Omega(x)$ is continuous in $S(d, \epsilon)$ outside $\gamma$ and is continuously extensible from the left and from the right to the part of $\gamma$ lying in $S(d, \epsilon)$. It follows from formula (7) that for any $x \in S(d, \epsilon)$ and $x \notin \gamma$ the inequality holds:

$$
|\mathcal{W}[\mu](x)| \leq \text { const. }
$$

Now we will study properties of derivatives of the double layer potential. It follows from Cauchy-Riemann relations that

$$
\frac{d \Phi(z)}{d z}=(\operatorname{Re} \Phi)_{x_{1}}^{\prime}-i(\operatorname{Re} \Phi)_{x_{2}}^{\prime}=-\mathcal{W}_{x_{1}}^{\prime}+i \mathcal{W}_{x_{2}}^{\prime}
$$

On the other hand, if $\mu(\sigma) \in C^{1, \lambda}[a, b]$, then for $z \notin \gamma$ :

$$
\begin{aligned}
\frac{d \Phi(z)}{d z} & =\frac{1}{2 \pi i} \int_{\gamma} \hat{\mu}(t) \frac{d}{d z} \frac{1}{t-z} d t=-\frac{1}{2 \pi i} \int_{\gamma} \hat{\mu}(t)\left(\frac{d}{d t} \frac{1}{t-z}\right) d t \\
& =-\frac{1}{2 \pi i}\left(\frac{\mu(b)}{t(b)-z}-\frac{\mu(a)}{t(a)-z}-\int_{\gamma} \frac{\hat{\mu}^{\prime}(t)}{t-z} d t\right)
\end{aligned}
$$

where

$$
\frac{d \hat{\mu}(t)}{d t}=\frac{d \mu(\sigma)}{d \sigma} \frac{d \sigma}{d t}=\frac{\mu^{\prime}(\sigma)}{t^{\prime}(\sigma)}=e^{-i \alpha(\sigma)} \mu^{\prime}(\sigma) .
$$


Since $\gamma \in C^{1, \lambda}$, then $e^{-i \alpha(\sigma)} \in C^{0, \lambda}[a, b]$, so one can show that $\hat{\mu}^{\prime}(t) \in C^{0, \lambda}(\gamma)$ (the proof repeats the above proof of the fact that $\hat{\mu}(t) \in C^{0, \lambda}(\gamma)$, if $\left.\mu(\sigma) \in C^{0, \lambda}[a, b]\right)$. From (9), (10) and from properties of the Cauchy-type integral [7, $\S 15]$, it follows that if $\mu(\sigma) \in C^{1, \lambda}[a, b]$, then $\nabla \mathcal{W}[\mu](x) \in C^{0}\left(\overline{R^{2} \backslash \gamma} \backslash X_{\gamma}\right)$; i.e., $\nabla \mathcal{W}[\mu](x)$ is continuously extensible to $\gamma$ from the left and from the right in interior points, though the limiting values of $\nabla \mathcal{W}[\mu](x)$ on $\gamma$ from the left and from the right can be different. We can write (10) in the form

$$
\frac{d \Phi(z)}{d z}=\frac{1}{2 \pi i}\left(\frac{\mu(b) e^{-i \psi(x, x(b))}}{|x-x(b)|}-\frac{\mu(a) e^{-i \psi(x, x(a))}}{|x-x(a)|}\right)+\Omega_{0}(z),
$$

where $\Omega_{0}(z)=\frac{1}{2 \pi i} \int_{\gamma} \frac{\hat{\mu}^{\prime}(t)}{t-z} d t$. It follows from [7, $\left.\S 22\right]$ that for all $z \in S(d, \epsilon)(d=a$ or $d=b)$, such that $z \notin \gamma$, the inequality holds:

$$
\left|\Omega_{0}(z)\right| \leq c_{0}\left(\left|\mu^{\prime}(d)\right| \ln \frac{1}{|x-x(d)|}+1\right) \leq c \ln \frac{1}{|x-x(d)|},
$$

where $c_{0}$ and $c$ are constants.

Comparing formulae (9) and (11), we obtain that for $x \in S(d, \epsilon)$ and $x \notin \gamma$, the formulae hold:

$$
\begin{aligned}
& \frac{\partial \mathcal{W}[\mu](x)}{\partial x_{1}}=\frac{1}{2 \pi} \frac{\mp \mu(d)}{|x-x(d)|} \sin \psi(x, x(d))+\Omega_{1}(x), \\
& \frac{\partial \mathcal{W}[\mu](x)}{\partial x_{2}}=\frac{1}{2 \pi} \frac{ \pm \mu(d)}{|x-x(d)|} \cos \psi(x, x(d))+\Omega_{2}(x),
\end{aligned}
$$

where

$$
\left|\Omega_{j}(x)\right| \leq c_{1}\left(\left|\mu^{\prime}(d)\right| \ln \frac{1}{|x-x(d)|}+1\right) \leq c_{2} \ln \frac{1}{|x-x(d)|}, \quad j=1,2,
$$

where $c_{1}, c_{2}$ are constants. The upper sign in the formulae is taken if $d=a$, while the lower sign is taken if $d=b$. It follows from $[7, \S 15.2]$ that if $\mu^{\prime}(d)=0$, then the functions $\Omega_{1}(x)$ and $\Omega_{2}(x)$ are continuously extensible to the end $x(d)$. Moreover, if $x \in S(d, \epsilon)$ and $x \notin \gamma$, then for the functions $\Omega_{1}(x)$ and $\Omega_{2}(x)$, the formulae hold:

$$
\begin{gathered}
\Omega_{1}(x)=-\operatorname{Re} \Omega_{0}(z) \\
=\mp \frac{\mu^{\prime}(d)}{2 \pi}\{\sin (\alpha(d)) \ln |x-x(d)|-\cos (\alpha(d)) \psi(x, x(d))\}+\Omega_{10}(x), \\
\Omega_{2}(x)=\operatorname{Im} \Omega_{0}(z) \\
= \pm \frac{\mu^{\prime}(d)}{2 \pi}\{\cos (\alpha(d)) \ln |x-x(d)|+\sin (\alpha(d)) \psi(x, x(d))\}+\Omega_{20}(x),
\end{gathered}
$$

which can be derived using the asymptotics for $\Omega_{0}(z)$ from $[7, \S 22]$. The upper sign in the formulae is taken if $d=a$, while the lower sign is taken if $d=b$. Functions $\Omega_{10}(x)$ and $\Omega_{20}(x)$ are continuously extensible to the end $x(d)$. By $\psi(x, x(d))$ we mean some fixed branch of this function, which varies continuously in $x$ in a neighbourhood of a point $x(d)$, slit along $\gamma$.

Let $\mu(\sigma) \in C^{1, \lambda}[a, b]$, and let $\mathbf{n}_{x}$ be a normal in the point $x \in \partial S(d, \epsilon)$, directed to the center of the circumference $\partial S(d, \epsilon)$, i.e., $\mathbf{n}_{x}=(-\cos \psi(x, x(d)),-\sin \psi(x, x(d)))$. Then 
we obtain from (12) for $x \notin \gamma$ :

$$
\left.\frac{\partial \mathcal{W}[\mu](x)}{\partial \mathbf{n}_{x}}\right|_{\partial S(d, \epsilon)}=-\Omega_{1}(x) \cos \psi(x, x(d))-\Omega_{2}(x) \sin \psi(x, x(d)) .
$$

Therefore, according to (13a):

$$
\left.\left|\frac{\partial \mathcal{W}[\mu](x)}{\partial \mathbf{n}_{x}}\right|\right|_{\partial S(d, \epsilon)} \leq\left.\operatorname{const} \ln \frac{1}{|x-x(d)|}\right|_{\partial S(d, \epsilon)}=\operatorname{const} \ln \frac{1}{\epsilon},
$$

since $|x-x(d)|=\epsilon$ on $\partial S(d, \epsilon)$. From this and from (8) we obtain that

$$
\begin{gathered}
\left|\int_{\partial S(d, \epsilon)} \mathcal{W}[\mu](x) \frac{\partial \mathcal{W}[\mu](x)}{\partial \mathbf{n}_{x}} d l\right| \leq \int_{0}^{2 \pi}|\mathcal{W}[\mu](x)| \cdot\left|\frac{\partial \mathcal{W}[\mu](x)}{\partial \mathbf{n}_{x}}\right| \epsilon d \psi \\
\leq 2 \pi \text { const } \epsilon \ln \frac{1}{\epsilon} \rightarrow 0
\end{gathered}
$$

if $\epsilon \rightarrow+0$, so

$$
\lim _{\epsilon \rightarrow+0} \int_{\partial S(d, \epsilon)} \mathcal{W}[\mu](x) \frac{\partial \mathcal{W}[\mu](x)}{\partial \mathbf{n}_{x}} d l=0 .
$$

Now let $\epsilon$ be a fixed positive number (sufficiently small). Using formulae (12) and setting $r=|x-x(d)|, \quad \psi=\psi(x, x(d))$, we consider the integral over the disc $S(d, \epsilon)$ :

$$
\begin{gathered}
\int_{S(d, \epsilon)}|\nabla \mathcal{W}[\mu](x)|^{2} d x=\int_{0}^{2 \pi} \int_{0}^{\epsilon}\left\{\left(\frac{\mu(d)}{2 \pi r}\right)^{2}\right. \\
\left.+\frac{\mu(d)}{\pi r}\left(\mp \Omega_{1}(x) \sin \psi \pm \Omega_{2}(x) \cos \psi\right)+\Omega_{1}^{2}(x)+\Omega_{2}^{2}(x)\right\} r d r d \psi=I_{1}+I_{2}, \\
I_{1}=\frac{1}{2 \pi} \int_{0}^{\epsilon} \frac{1}{r} \mu^{2}(d) d r, \\
I_{2}=\int_{0}^{2 \pi} \int_{0}^{\epsilon}\left\{\frac{\mu(d)}{\pi}\left(\mp \Omega_{1}(x) \sin \psi \pm \Omega_{2}(x) \cos \psi\right)+r\left(\Omega_{1}^{2}(x)+\Omega_{2}^{2}(x)\right)\right\} d r d \psi .
\end{gathered}
$$

The integral $I_{2}$ converges according to estinates (13a):

$$
\left|I_{2}\right| \leq 4 c_{2} \int_{0}^{\epsilon} \ln \frac{1}{r}\left(|\mu(d)|+c_{2} \pi r \ln \frac{1}{r}\right) d r \leq \text { const. }
$$

Hence, if integral (15) converges, then the integral $I_{1}$ converges as well (as a difference of two convergent integrals), but the integral $I_{1}$ converges if and only if $\mu(d)=0$, while in other cases $I_{1}$ diverges. Thus, the integral (15) converges if and only if $\mu(d)=0$. Consequently $|\nabla \mathcal{W}[\mu](x)|$ belongs to $L_{2}(S(d, \epsilon))$ with small $\epsilon>0$ if and only if $\mu(d)=0$. Let us formulate obtained results in the form of the theorem.

TheOrem 2. Let $\gamma$ be an open curve of class $C^{1, \lambda}, \lambda \in(0,1]$. Let $S(d, \epsilon)$ be a disc of a sufficiently small radius $\epsilon$ with the center in the point $x(d) \in X_{\gamma} \quad(d=a$ or $d=b)$.

I. If $\mu(s) \in C^{0, \lambda}[a, b]$, then $\mathcal{W}[\mu](x) \in C^{0}\left(\overline{R^{2} \backslash \gamma} \backslash X_{\gamma}\right)$ and for any $x \in S(d, \epsilon)$, such that $x \notin \gamma$, the relationships (7), (8) hold.

II. If $\mu(s) \in C^{1, \lambda}[a, b]$, then

1) $\nabla \mathcal{W}[\mu](x) \in C^{0}\left(\overline{R^{2} \backslash \gamma} \backslash X_{\gamma}\right)$; 
2) for any $x \in S(d, \epsilon)$, such that $x \notin \gamma$, the formulae (12) hold, in which the functions $\Omega_{1}(x)$ and $\Omega_{2}(x)$ satisfy relationships (13);

3) for $\mathcal{W}[\mu](x)$ the property (14) holds;

4) $|\nabla \mathcal{W}[\mu](x)|$ belongs to $L_{2}(S(d, \epsilon))$ for sufficiently small $\epsilon>0$ if and only if $\mu(d)=0$.

REMARK. Each function of class $C^{0}\left(\overline{R^{2} \backslash \gamma} \backslash X_{\gamma}\right)$ is continuous in $R^{2} \backslash \gamma$, is continuously extensible to $\gamma \backslash X_{\gamma}$ from the left and from the right, but limiting values of such a function on $\gamma \backslash X_{\gamma}$ from the left and from the right can be different, i.e., the function may have a jump on $\gamma \backslash X_{\gamma}$.

Let us study smoothness of the direct value of the double layer potential on the curve.

Lemma. Let $\gamma$ be an open curve of class $C^{2, \lambda}, \lambda \in(0,1]$, and let $\mu(s) \in C^{0}[a, b]$. Let

$$
I_{1}(s)=-\frac{1}{2 \pi} \int_{\gamma} \mu(\sigma) \frac{\partial \ln |x(s)-y(\sigma)|}{\partial \mathbf{n}_{y}} d \sigma
$$

be the direct value of the double layer potential $\mathcal{W}[\mu](x)$ on $\gamma$. Then

$$
I_{1}(s) \in C^{1, \lambda / 4}[a, b] .
$$

Proof. Let us prove that $I_{1}(s) \in C^{1, \lambda / 4}[a, b]$. Taking into account that $\mathbf{n}_{y}=\left(y_{2}^{\prime}(\sigma),-y_{1}^{\prime}(\sigma)\right)$, we find

$$
\begin{gathered}
\frac{\partial \ln |x(s)-y(\sigma)|}{\partial \mathbf{n}_{y}}=\frac{T(s, \sigma)}{g(s, \sigma)}, \quad g(s, \sigma)=\frac{|x(s)-y(\sigma)|^{2}}{(s-\sigma)^{2}}, \\
T(s, \sigma)=\frac{\left[x_{2}(s)-y_{2}(\sigma)\right] y_{1}^{\prime}(\sigma)-\left[x_{1}(s)-y_{1}(\sigma)\right] y_{2}^{\prime}(\sigma)}{(s-\sigma)^{2}} .
\end{gathered}
$$

Note that $y(\sigma)$ is a point on $\Gamma$ corresponding to $s=\sigma$. So, we may put $x(\sigma)=y(\sigma)$. For $j=1,2$ we have $[11, \S 3]$

$$
x_{j}(s)-x_{j}(\sigma)=(s-\sigma) Z_{j}^{1}(s, \sigma)=-x_{j}^{\prime}(\sigma)(\sigma-s)+(\sigma-s)^{2} Z_{j}^{2}(\sigma, s),
$$

where

$$
\begin{aligned}
& Z_{j}^{1}(s, \sigma)=\int_{0}^{1} x_{j}^{\prime}(\sigma+\xi(s-\sigma)) d \xi \in C^{1, \lambda}([a, b] \times[a, b]), \\
& Z_{j}^{2}(\sigma, s)=\int_{0}^{1} \xi x_{j}^{\prime \prime}(s+\xi(\sigma-s)) d \xi \in C^{0, \lambda}([a, b] \times[a, b]) .
\end{aligned}
$$

Note that the function

$$
g(s, \sigma)=\frac{|x(s)-x(\sigma)|^{2}}{(s-\sigma)^{2}}=\left\{\left[Z_{1}^{1}(s, \sigma)\right]^{2}+\left[Z_{2}^{1}(s, \sigma)\right]^{2}\right\} \in C^{1, \lambda}([a, b] \times[a, b])
$$

does not equal zero anywhere on $\Gamma$ and $g(s, s)=1$. Therefore

$$
\frac{1}{g(s, \sigma)} \in C^{1}([a, b] \times[a, b]) .
$$


Further,

$$
\begin{gathered}
\frac{\partial}{\partial s} \frac{1}{g(s, \sigma)}=\frac{\partial}{\partial s} \frac{(s-\sigma)^{2}}{|x(s)-x(\sigma)|^{2}}=-\frac{g_{s}^{\prime}(s, \sigma)}{g^{2}(s, \sigma)} \\
=-2 \frac{Z_{1}^{1}(s, \sigma)\left[Z_{1}^{1}(s, \sigma)\right]_{s}^{\prime}+Z_{2}^{1}(s, \sigma)\left[Z_{2}^{1}(s, \sigma)\right]_{s}^{\prime}}{g^{2}(s, \sigma)} \in C^{0, \lambda}([a, b] \times[a, b]) .
\end{gathered}
$$

Consequently, $\frac{1}{g(s, \sigma)} \in C^{1, \lambda}([a, b] \times[a, b])$. Similarly,

$$
\begin{aligned}
T(s, \sigma) & =\frac{\left[x_{2}(s)-x_{2}(\sigma)\right] x_{1}^{\prime}(\sigma)-\left[x_{1}(s)-x_{1}(\sigma)\right] x_{2}^{\prime}(\sigma)}{(s-\sigma)^{2}} \\
& =\left[Z_{2}^{2}(\sigma, s) x_{1}^{\prime}(\sigma)-Z_{1}^{2}(\sigma, s) x_{2}^{\prime}(\sigma)\right] \in C^{0, \lambda}([a, b] \times[a, b]) .
\end{aligned}
$$

Consider $\frac{\partial T(s, \sigma)}{\partial s}=J_{1}(s, \sigma)-2 J_{2}(s, \sigma)$, where

$$
\begin{aligned}
J_{1}(s, \sigma) & =\frac{x_{2}^{\prime}(s) x_{1}^{\prime}(\sigma)-x_{1}^{\prime}(s) x_{2}^{\prime}(\sigma)}{(s-\sigma)^{2}} \\
& =\frac{\left[x_{2}^{\prime}(s)-x_{2}^{\prime}(\sigma)\right] x_{1}^{\prime}(\sigma)-\left[x_{1}^{\prime}(s)-x_{1}^{\prime}(\sigma)\right] x_{2}^{\prime}(\sigma)}{(s-\sigma)^{2}} \\
& =\frac{1}{s-\sigma}\left\{x_{1}^{\prime}(\sigma) \int_{0}^{1} x_{2}^{\prime \prime}[s+\xi(\sigma-s)] d \xi\right. \\
& \left.-x_{2}^{\prime}(\sigma) \int_{0}^{1} x_{1}^{\prime \prime}[s+\xi(\sigma-s)] d \xi\right\} ; \\
J_{2}(s, \sigma) & =\frac{\left[x_{2}(s)-x_{2}(\sigma)\right] x_{1}^{\prime}(\sigma)-\left[x_{1}(s)-x_{1}(\sigma)\right] x_{2}^{\prime}(\sigma)}{(s-\sigma)^{3}} \\
& =\frac{1}{s-\sigma}\left\{x_{1}^{\prime}(\sigma) \int_{0}^{1} \xi x_{2}^{\prime \prime}[s+\xi(\sigma-s)] d \xi\right. \\
& \left.-x_{2}^{\prime}(\sigma) \int_{0}^{1} \xi x_{1}^{\prime \prime}[s+\xi(\sigma-s)] d \xi\right\} .
\end{aligned}
$$

Then

$$
\begin{aligned}
\frac{\partial T(s, \sigma)}{\partial s}= & \frac{1}{s-\sigma}\left\{x_{1}^{\prime}(\sigma) \int_{0}^{1}(1-2 \xi) x_{2}^{\prime \prime}[s+\xi(\sigma-s)] d \xi\right. \\
& \left.-x_{2}^{\prime}(\sigma) \int_{0}^{1}(1-2 \xi) x_{1}^{\prime \prime}[s+\xi(\sigma-s)] d \xi\right\}=\frac{K(s, \sigma)}{s-\sigma},
\end{aligned}
$$

where $K(s, \sigma) \in C^{0, \lambda}([a, b] \times[a, b])$ and $K(s, s)=0$. According to [7, $\left.\S 5.7\right]$, the following representation holds:

$$
\frac{\partial T(s, \sigma)}{\partial s}=\frac{K^{*}(s, \sigma)}{|s-\sigma|^{1-\lambda / 4}}
$$


where $K^{*}(s, \sigma) \in C^{0,3 \lambda / 4}([a, b] \times[a, b])$. Using properties of Hölder functions [7], we obtain the representation

$$
\begin{aligned}
\frac{\partial}{\partial s} \frac{\partial \ln |x(s)-y(\sigma)|}{\partial \mathbf{n}_{y}} & =\frac{1}{g(s, \sigma)} \frac{\partial T(s, \sigma)}{\partial s}+T(s, \sigma) \frac{\partial}{\partial s} \frac{1}{g(s, \sigma)} \\
& =\frac{K_{1}(s, \sigma)}{|s-\sigma|^{1-\lambda / 4}}+K_{2}(s, \sigma),
\end{aligned}
$$

where $K_{1}(s, \sigma) \in C^{0,3 \lambda / 4}([a, b] \times[a, b]), \quad K_{2}(s, \sigma) \in C^{0, \lambda}([a, b] \times[a, b])$. By formal differentiation under the integral, we find

$$
\begin{aligned}
\frac{d I_{1}(s)}{d s} & =-\frac{1}{2 \pi} \int_{\gamma} \mu(\sigma) \frac{\partial}{\partial s} \frac{\partial \ln |x(s)-y(\sigma)|}{\partial \mathbf{n}_{y}} d \sigma \\
& =-\frac{1}{2 \pi} \int_{\gamma} \mu(\sigma) \frac{K_{1}(s, \sigma)}{|s-\sigma|^{1-\lambda / 4}} d \sigma-\frac{1}{2 \pi} \int_{\gamma} \mu(\sigma) K_{2}(s, \sigma) d \sigma .
\end{aligned}
$$

The validity of differentiation under the integral can be proved in the same way as at the end of $\S 1.6$ in [9] (Fubini's theorem on change of integration order is used). Taking into account the obtained representation for $\frac{d I_{1}(s)}{d s}$ and applying the results of $[7, \S 51.1]$, we obtain that $\frac{d I_{1}(s)}{d s} \in C^{0, \lambda / 4}[a, b]$. The lemma is proved.

4. Existence of a classical solution and non-existence of a weak solution. We will construct the solution to the problem $\mathbf{D}_{1}$ with the assumption that $F^{+}(s), F^{-}(s) \in C^{1, \lambda}\left(\Gamma^{1}\right), \quad \lambda \in(0,1], \quad F(s) \in C^{0}\left(\Gamma^{2}\right)$. We will look for a solution to the problem $\mathbf{D}_{1}$ in the form

$$
u(x)=-w\left[F^{+}-F^{-}\right](x)+v(x),
$$

where

$$
w\left[F^{+}-F^{-}\right](x)=-\frac{1}{2 \pi} \int_{\Gamma^{1}}\left(F^{+}(\sigma)-F^{-}(\sigma)\right) \frac{\partial}{\partial \mathbf{n}_{y}} \ln |x-y(\sigma)| d \sigma
$$

is the double layer potential. The potential $w\left[F^{+}-F^{-}\right](x)$ satisfies the Laplace equation (2a) in $\mathcal{D} \backslash \Gamma^{1}$ and belongs to the class $\mathbf{K}_{1}$ according to Theorem 2. Limiting values of the potential $w\left[F^{+}-F^{-}\right](x)$ on $\left(\Gamma^{1}\right)^{ \pm}$are given by the formula

$$
\left.w\left[F^{+}-F^{-}\right](x)\right|_{x(s) \in\left(\Gamma^{1}\right)^{ \pm}}=\mp\left(F^{+}(s)-F^{-}(s)\right) / 2+w\left[F^{+}-F^{-}\right](x(s)),
$$

where $w\left[F^{+}-F^{-}\right](x(s))$ is the direct value of the potential on $\Gamma^{1}$.

The function $v(x)$ in (16) must be a solution to the following problem.

Problem D. Find a function $v(x) \in C^{0}(\overline{\mathcal{D}}) \cap C^{2}\left(\mathcal{D} \backslash \Gamma^{1}\right)$ that obeys the Laplace equation (2a) in the domain $\mathcal{D} \backslash \Gamma^{1}$ and satisfies the boundary conditions

$$
\begin{gathered}
\left.v(x)\right|_{x(s) \in \Gamma^{1}}=\left(F^{+}(s)+F^{-}(s)\right) / 2+w\left[F^{+}-F^{-}\right](x(s))=f(s), \\
\left.v(x)\right|_{x(s) \in \Gamma^{2}}=F(s)+w\left[F^{+}-F^{-}\right](x(s))=f(s) .
\end{gathered}
$$

(If $x(s) \in \Gamma^{1}$, then $w\left[F^{+}-F^{-}\right](x(s))$ is the direct value of the potential on $\Gamma^{1}$.) 
If $\mathcal{D}$ is an exterior domain, then we add the following condition at infinity:

$$
|v(x)| \leq \text { const }, \quad|x|=\sqrt{x_{1}^{2}+x_{2}^{2}} \rightarrow \infty .
$$

All conditions of the problem $\mathcal{D}$ have to be satisfied in a classical sense. Obviously, $w\left[F^{+}-F^{-}\right](x(s)) \in C^{0}\left(\Gamma^{2}\right)$. It follows from the lemma that $w\left[F^{+}-F^{-}\right](x(s)) \in C^{1, \lambda / 4}\left(\Gamma^{1}\right)$ (here by $w\left[F^{+}-F^{-}\right](x(s))$ we mean the direct value of the potential on $\left.\Gamma^{1}\right)$. So, $f(s) \in C^{1, \lambda / 4}\left(\Gamma^{1}\right)$ and $f(s) \in C^{0}\left(\Gamma^{2}\right)$.

We will look for the function $v(x)$ in the smoothness class $\mathbf{K}$.

We say that the function $v(x)$ belongs to the smoothness class $\mathbf{K}$ if

(1) $v(x) \in C^{0}(\overline{\mathcal{D}}) \cap C^{2}\left(\mathcal{D} \backslash \Gamma^{1}\right), \quad \nabla v \in C^{0}\left(\overline{\mathcal{D} \backslash \Gamma^{1}} \backslash \Gamma^{2} \backslash X\right)$, where $X$ is a pointset consisting of the endpoints of $\Gamma^{1}$;

(2) in a neighbourhood of any point $x(d) \in X$ for some constants $\mathcal{C}>0, \delta>-1$ the inequality $|\nabla v| \leq \mathcal{C}|x-x(d)|^{\delta}$ holds, where $x \rightarrow x(d)$ and $d=a_{n}^{1}$ or $d=b_{n}^{1}$, $n=1, \ldots, N_{1}$.

The definition of the functional class $C^{0}\left(\overline{\mathcal{D} \backslash \Gamma^{1}} \backslash \Gamma^{2} \backslash X\right)$ is given in the remark to the definition of the smoothness class $\mathbf{K}_{1}$. Clearly, $\mathbf{K} \subset \mathbf{K}_{1}$; i.e., if $v(x) \in \mathbf{K}$, then $v(x) \in \mathbf{K}_{1}$.

It can be verified directly that if $v(x)$ is a solution to the problem $\mathbf{D}$ in the class $\mathbf{K}$, then the function (16) is a solution to the problem $\mathbf{D}_{1}$.

Theorem 3. Let $\Gamma \in C^{2, \lambda / 4}, \quad f(s) \in C^{1, \lambda / 4}\left(\Gamma^{1}\right), \lambda \in(0,1], \quad f(s) \in C^{0}\left(\Gamma^{2}\right)$. Then the solution to the problem $\mathbf{D}$ in the smoothness class $\mathbf{K}$ exists and is unique.

Theorem 3 has been proved in the following papers:

1 ) in $[2,3]$, if $\mathcal{D}$ is an interior domain;

2) in [4], if $\mathcal{D}$ is an exterior domain and $\Gamma^{2} \neq \emptyset$;

3 ) in $[5,6]$, if $\Gamma^{2}=\emptyset$ and so $\mathcal{D}=R^{2}$ is an exterior domain.

In all of these papers, the integral representations for the solution to the problem $\mathbf{D}$ in the class $\mathbf{K}$ are obtained in the form of potentials, densities in which are defined from the uniquely solvable Fredholm integro-algebraic equations of the second kind and index zero. Uniqueness of a solution to the problem $\mathbf{D}$ is proved either by the maximum principle or by the method of energy (integral) identities. In the latter case we take into account that a solution to the problem belongs to the class $\mathbf{K}$. Note that the problem $\mathbf{D}$ is a particular case of more general boundary value problems studied in [3]-[6].

Note that conditions of Theorem 3 hold if $\Gamma \in C^{2, \lambda}, F^{+}(s), F^{-}(s) \in C^{1, \lambda}\left(\Gamma^{1}\right)$, $\lambda \in(0,1], F(s) \in C^{0}\left(\Gamma^{2}\right)$. From Theorems 2, 3 we obtain the solvability of the problem $\mathbf{D}_{1}$.

Theorem 4. Let $\Gamma \in C^{2, \lambda}, \quad F^{+}(s), F^{-}(s) \in C^{1, \lambda}\left(\Gamma^{1}\right), \quad \lambda \in(0,1], \quad F(s) \in C^{0}\left(\Gamma^{2}\right)$. Then a solution to the problem $\mathbf{D}_{1}$ exists and is given by the formula (16), where $v(x)$ is a unique solution to the problem $\mathbf{D}$ in the class $\mathbf{K}$, ensured by Theorem 3.

REMARK. Let us check that the solution to the problem $\mathbf{D}_{1}$ given by formula (16) satisfies condition (1). Let $d=a_{n}^{1}$ or $d=b_{n}^{1} \quad\left(n=1, \ldots, N_{1}\right)$ and let $r$ be small enough. 
Then substituting (16) in the integral in (1) we obtain

$$
\begin{gathered}
\int_{\partial S(d, r)} u(x) \frac{\partial u(x)}{\partial \mathbf{n}_{x}} d l \\
=\int_{\partial S(d, r)} w(x) \frac{\partial w(x)}{\partial \mathbf{n}_{x}} d l-\int_{\partial S(d, r)} w(x) \frac{\partial v(x)}{\partial \mathbf{n}_{x}} d l-\int_{\partial S(d, r)} v(x) \frac{\partial w(x)}{\partial \mathbf{n}_{x}} d l+\int_{\partial S(d, r)} v(x) \frac{\partial v(x)}{\partial \mathbf{n}_{x}} d l .
\end{gathered}
$$

If $r \rightarrow 0$, then the first term tends to zero by Theorem 2(II.3). As mentioned above, $v(x) \in \mathbf{K} \subset \mathbf{K}_{1}$; therefore condition (1) holds for the function $v(x)$, so the fourth term tends to zero as $r \rightarrow 0$. The second term tends to zero as $r \rightarrow 0$, since $w(x)$ is bounded at the ends of $\Gamma^{1}$ according to Theorem 2(I), and since $v(x)$ satisfies condition 2) in the definition of the class $\mathbf{K}$. Noting that $v(x)$ is continuous at the ends of $\Gamma^{1}$ owing to the definition of the class $\mathbf{K}$, and using Theorem 2(II.2) for calculation of $\frac{\partial w(x)}{\partial \mathbf{n}_{x}}$ in the third term, we deduce that the third term tends to zero when $r \rightarrow 0$ as well. Consequently, equality (1) holds for the solution to the problem $\mathbf{D}_{1}$ constructed in Theorem 4 .

Uniqueness of a solution to the problem $\mathbf{D}_{1}$ follows from Theorem 1 . In fact, the solution to the problem $\mathbf{D}_{1}$ found in Theorem 4 is a classical solution. Let us discuss under which conditions this solution to the problem $\mathbf{D}_{1}$ is not a weak solution. Let $u(x)$ be a solution to the problem $\mathbf{D}_{1}$ defined in Theorem 4 by the formula (16). Consider a disc $S(d, \epsilon)$ with the center in the point $x(d) \in X$ and of radius $\epsilon>0 \quad\left(d=a_{n}^{1}\right.$ or $\left.d=b_{n}^{1}, \quad n=1, \ldots, N_{1}\right)$. In doing so, $\epsilon$ is a fixed positive number, which can be taken small enough. Since $v(x) \in \mathbf{K}$, we have $v(x) \in L_{2}(S(d, \epsilon))$ and $|\nabla v(x)| \in L_{2}(S(d, \epsilon))$ (this follows from the definition of the smoothness class $\mathbf{K}$ ). Let $x \in S(d, \epsilon)$ and $x \notin \Gamma^{1}$. It follows from (16) that $|\nabla w[\mu](x)| \leq|\nabla u(x)|+|\nabla v(x)|$, whence

$$
|\nabla w[\mu](x)|^{2} \leq|\nabla u(x)|^{2}+|\nabla v(x)|^{2}+2|\nabla u(x)| \cdot|\nabla v(x)| \leq 2\left(|\nabla u(x)|^{2}+|\nabla v(x)|^{2}\right),
$$

since $2|\nabla u(x)| \cdot|\nabla v(x)| \leq|\nabla u(x)|^{2}+|\nabla v(x)|^{2}$. Assume that $|\nabla u(x)|$ belongs to $L_{2}(S(d, \epsilon))$. Then, integrating this inequality over $S(d, \epsilon)$, we obtain $\left.\|\nabla w\|^{2}\right|_{L_{2}(S(d, \epsilon))} \leq 2\left(\left.\|\nabla u\|^{2}\right|_{L_{2}(S(d, \epsilon))}+\left.\|\nabla v\|^{2}\right|_{L_{2}(S(d, \epsilon))}\right)$. Consequently, if $|\nabla u(x)| \epsilon$ $L_{2}(S(d, \epsilon))$, then $|\nabla w| \in L_{2}(S(d, \epsilon))$. However, according to Theorem 2, if $F^{+}(d)-$ $F^{-}(d) \neq 0$, then $|\nabla w|$ does not belong to $L_{2}(S(d, \epsilon))$. Therefore, if $F^{+}(d) \neq F^{-}(d)$, then our assumption that $|\nabla u| \in L_{2}(S(d, \epsilon))$ does not hold; i.e., $|\nabla u| \notin L_{2}(S(d, \epsilon))$. Thus, if among numbers $a_{1}^{1}, \ldots, a_{N_{1}}^{1}, b_{1}^{1}, \ldots, b_{N_{1}}^{1}$ there exists such a number $d$ that $F^{+}(d) \neq F^{-}(d)$, then for some $\epsilon>0$ we have $|\nabla u| \notin L_{2}(S(d, \epsilon))=L_{2}\left(S(d, \epsilon) \backslash \Gamma^{1}\right)$, so $u \notin W_{2}^{1}\left(S(d, \epsilon) \backslash \Gamma^{1}\right)$, where $W_{2}^{1}$ is a Sobolev space of functions from $L_{2}$, which have generalized derivatives from $L_{2}$. We have proved

THEOREM 5. Let conditions of Theorem 4 hold and among numbers $a_{1}^{1}, . ., a_{N_{1}}^{1}, b_{1}^{1}, \ldots, b_{N_{1}}^{1}$ there exists such a number $d$, that $F^{+}(d) \neq F^{-}(d)$. Then the solution to the problem $\mathbf{D}_{1}$, ensured by Theorem 4, does not belong to $W_{2}^{1}\left(S(d, \epsilon) \backslash \Gamma^{1}\right)$ for some $\epsilon>0$, whence it follows that it does not belong to $W_{2}^{1}\left(\mathcal{D} \backslash \Gamma^{1}\right)$. Here $S(d, \epsilon)$ is a disc of a radius $\epsilon$ with the center in the point $x(d) \in X$. 
If conditions of Theorem 5 hold, then the unique solution to the problem $\mathbf{D}_{1}$, constructed in Theorem 4, does not belong to $W_{2}^{1}\left(\mathcal{D} \backslash \Gamma^{1}\right)$, and so it is not a weak solution. We arrive at the following

Corollary. Let conditions of Theorem 5 hold. Then a weak solution to the problem $\mathbf{D}_{1}$ in the space $W_{2}^{1}\left(\mathcal{D} \backslash \Gamma^{1}\right)$ does not exist.

Remark. Even if the number $d$, mentioned in Theorem 5, does not exist, then the solution $u(x)$ to the problem $\mathbf{D}_{1}$, ensured by Theorem 4 , may not be a weak solution to the problem $\mathbf{D}_{1}$. This happens, for instance, in the case when $\mathcal{D}$ is an exterior domain and if $\lim _{|x| \rightarrow \infty} u(x) \neq 0$, whence it follows that $u \notin L_{2}\left(\mathcal{D} \backslash \Gamma^{1}\right)$, and so $u \notin W_{2}^{1}\left(\mathcal{D} \backslash \Gamma^{1}\right)$. In this case a weak solution to the problem $\mathbf{D}_{1}$ in $W_{2}^{1}\left(\mathcal{D} \backslash \Gamma^{1}\right)$ does not exist also.

Clearly, $L_{2}\left(\mathcal{D} \backslash \Gamma^{1}\right)=L_{2}(\mathcal{D})$, since $\Gamma^{1}$ is a set of zero measure.

This research has been partially supported by the RFBR grant 05-01-00050.

\section{REFERENCES}

[1] Lifanov I. K. Method of singular integral equations and discrete vortices. — VSP, Zeist, 1996. MR1451377 (98g:65130)

[2] Krutitskii P.A. The integral representation for a solution of the 2-D Dirichlet problem with boundary data on closed and open curves. Mathematika (London), 2000, v.47, p.339-354. MR1924510 (2003f:35037)

[3] Krutitskii P.A. The Dirichlet problem for the 2-D Laplace equation in a multiply connected domain with cuts. Proc. Edinburgh Math. Soc., 2000, v.43, p.325-341. MR1763054 (2002h:31002)

[4] Krutitskii P.A. The 2-dimensional Dirichlet problem in an external domain with cuts. Zeitschr. Analysis u. Anwend., 1998, v.17, No.2, p.361-378. MR1632551 (99f:35036)

[5] Krutitskii P.A. The mixed problem for the Laplace equation outside cuts in a plane. Differential Equations, 1997, v.33, pp.1184-1193. MR1638915 (99h:35030)

[6] Krutitskii P.A. The mixed problem in an exterior cracked domain with Dirichlet condition on cracks. Computers and Mathematics with Applications, 2005, v.50, p.769-782. MR2165638 (2006c:35063)

[7] Muskhelishvili N.I. Singular integral equations. Nauka, Moscow, 1968. (In Russian; English translation: Noordhoff, Groningen, 1972.) MR0355494 (50:7968)

[8] Smirnov V. I. A course of higher mathematics. V. IV, V. Pergamon Press, Oxford, 1964.

[9] Vladimirov V.S. Equations of mathematical physics. Nauka, Moscow, 1981. (In Russian, English translation: Mir Publishers, Moscow, 1984.) MR764399 (86f:00030)

[10] Gilbarg D., Trudinger N.S. Elliptic partial differential equations of second order. Springer-Verlag, Berlin-New York, 1977. MR0473443 (57:13109)

[11] Krutitskii P. A. The Dirichlet problem for the Helmholtz equation in the exterior of cuts in the plane. Comput. Maths. Math. Phys., 1994, v.34, No.8/9, pp.1073-1090. MR1300397 (95f:35046) 